\title{
Transit Bus Route Network Design: a model and its application in a real network
}

\author{
B. Barabino \\ Department of Land Engineering, Cagliari University, Italy
}

\begin{abstract}
This paper deals with Transit Bus Route Network Design (TBRND) and proposes a new heuristic model for its resolution. Planning a bus network is a coordinate and logical process that leads to the definition of routes, frequencies, timetable plans, vehicles and bus driver scheduling. Routes and frequencies are the most important phases of the planning. Therefore, a model defining a complex objective function has been structured to determine routes and frequencies. This model considers many interesting parameters related to the public transportation network. For its resolution, a complex two-phase heuristic algorithm has been used. In the first one, the route lines are characterized whilst in the second, the frequencies are determined, respecting geometric, operative and congruence constraints. Greedy resolution techniques were employed in the first part, while the second part of the algorithm has been resolved through a random optimization process. The method was implemented and resolved using JAVA language. This model has been experimented on a medium-sized city in Italy, and it has provided some interesting results that make the adopted procedure valid in comparison to the existing network. In particular, they proved effective with specific regard to the business service, standing out for shorter distances covered by the lines, and a $22 \%$ reduction of the total travel time against the present scenario.
\end{abstract}

Keywords: Bus, network design, heuristic, land use.

\section{Introduction}

Public transport has been widely recognized as an effective method towards the improvement of people's mobility in terms of energetic, urban space saving, and external costs reduction [1]. Therefore, planning an efficient bus network is 
important for the social, economic and physical structure of a city. Planning a bus network consists of a coordinate and logical process articulated in five phases: the definition of routes, frequencies, timetable plans and bus and driver scheduling. However, the most important activities can be identified in the first and second phase. Once these milestones are set, it is possible to determine the remaining parts with good approximation $[2,3]$. Generally, approaches used to resolve Transit Bus Route Network Design (TBRND) problem can be classified into empirical, what if and what to [4-6]. Empirical methods consist mainly of a qualitative approach, based on the experience of the planner or on the indications supplied by control organisations [7]. The generated networks are not subject to simulation tests. What if methods are usually based on the experience of the planner, who proposes solutions that are tested by simulating the system and evaluating its characteristics $[8,9]$. What methods represent the third approach and consist of formulating the network planning problem as a restricted optimization problem with a specific objective function. What models can be classified into analytical and heuristic. The former use simplified networks to determine the optimal configurations of some public transport parameters, creating optimal networks not influenced by the current scenario, but limited in the number of elements $[10,11]$. The latter bridges the gap between simulation techniques and analytical optimisation systems. They use automatic procedures to generate and to improve the transportation networks, while the complexity of the problem is resolved breaking it down into more manageable parts. Some authors employ this approach [12-27]. In this context, this paper presents a new heuristic model for the TBRND applied to an Italian real case.

\section{The model: formulation and resolution}

Given a determined period of the day, the problem is to decide, among a certain number of routes and frequencies, which system provides the best overall travel time performance. This is done on the basis of geometric, exercise and financial considerations. The proposed model deals with making a weighed cost function explicit, which takes into account the requirements of the two main actors involved in the planning process: the company and the user. The decisional variables are represented by the routes followed by the bus (ITI), the service frequency (f) and the bus dimensions (d). At a descriptive level it deals with the passengers flows, on which it is not possible to act directly, but only indirectly through a variation of the supply system (e.g. varying the frequencies) since it is calculated through the hyper-path assignment procedure [28]. Three types of constraints are included. The chosen model is static with a fixed demand.

\subsection{Specification of the objective function and the constraints}

The objective is the minimisation of the cost of operator and user for a given transportation demand. As a result, the objective function has the form of eqn. (1): 


$$
\begin{aligned}
& \min =C_{a}+C_{u}=
\end{aligned}
$$

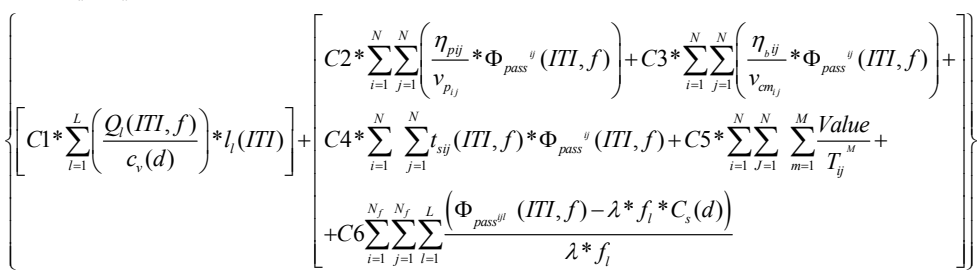

s.t:

$$
\begin{aligned}
& L_{C} \leq M L \\
& L_{\text {min }}<l_{l}<L_{\text {max }} \quad \forall l \in L \\
& \tau_{\min }<\tau_{l}<\tau_{\max } \quad \forall l \in L \\
& \tau_{\max }^{l} \leq W^{*} \tau_{\min }^{l} \quad \forall l \in L \\
& D_{i, j}^{a} \leq D_{a \max } \quad \forall i \in N_{p} \text { e } \forall j \in N_{f} \\
& D_{i, j}^{f} \leq D_{f \max } \quad \forall i, j \in N_{f} \\
& \sum_{i=1}^{N} \sum_{j=1}^{N} \sum_{l=1}^{L}\left(\frac{p_{i j}^{l}}{P}\right) \geq \% P \\
& f_{\text {min }} \leq f_{l} \leq f_{\max } \quad \forall l \in L, \forall \text { f integer } \\
& \left(\frac{Q_{l}}{c_{v}(d)^{*} f_{l}}\right) \leq L F \quad \forall l \in L \\
& \sum_{l=1}^{L}\left(\left(\tau_{g}^{l}+\tau_{s}\right)^{*} f_{l}\right) \leq N_{\max } \\
& \mathrm{d}_{\mathrm{hl}}=0 / 1 \quad \forall l \in L, \forall h \in d \\
& t_{m}^{\operatorname{Min}} \leq t_{m} \leq t_{m}^{\operatorname{Max}} \quad \forall m \in M \\
& \sum_{j, i \in B S(i)} \Phi_{\text {pass }}^{k}{ }_{j i}^{k}-\sum_{i, j \in F S(i)} \Phi_{\text {pass }_{i j}}^{k}= \pm P i^{k} \quad \forall i \in N_{C} \mathrm{e}^{\forall k \in(O, D)} \\
& \sum_{j, i \in B S(i)} \Phi_{\text {pass }}^{k} \underset{j i}{k}-\sum_{i, j \in F S(i)} \Phi_{\text {pass }_{i j}}^{k}=0 \quad \forall i, N \neq N_{\mathcal{C}} \text { e } \forall k \in(O, D) \\
& \Phi_{\text {pass }_{i j}}^{l}>0 \quad \forall i, j \in N, \forall l \in L
\end{aligned}
$$

where:

- $\quad N$ is the overall set of network nodes ;

- $N_{f}$ identifies the bus stop nodes set;

- $\quad \mathrm{i}, \mathrm{j}$ represent the extreme nodes of a generic link;

- $\quad M$ is the mode set on the network;

- L denotes the line network sets ; 
- $\mathrm{Q}_{1}$ is max. passenger flows on the line 1 ;

- $\quad \mathrm{c}_{\mathrm{v}}(\mathrm{d})$ is the vehicle capacity - of d dimension- used in the line;

- $\mathrm{l}_{1}$ is the length of the corresponding line;

- $\eta_{p_{i j}}$ is the length of the (i,j) walk link;

- $\quad \mathrm{v}_{\mathrm{pij}}$ is the pedestrian speed;

- $\quad \eta_{b_{i j}}$ is the length of the on board link;

- $\quad \mathrm{v}_{\mathrm{cmij}}$ is the vehicle commercial speed;

- $t_{s i j}(f)$ is the user wait time at the bus stop;

- Value is the economic value of an hour for the user;

- $\mathrm{T}_{\mathrm{ij}}{ }^{\mathrm{M}}$ is the cost associated to the boarding link of the fare paid by the user from node $i$ to $j$ on mode $\mathrm{m}$ of $\mathrm{M}$;

- $\lambda$ is the length of reference time period;

- $C_{s}(\mathrm{~d})$ is the vehicle seating capacity;

- $\mathrm{C}_{1} \ldots \mathrm{C}_{6}$ represents cost coefficients;

- $\mathrm{L}_{\mathrm{C}}$ is total network lines number;

- $\quad \mathrm{ML}$ is the max. number of network lines;

- $\mathrm{L}_{1}$ is the length of a generic line 1 ;

- $\mathrm{L}_{\min }$ and $\mathrm{L}_{\max }$ are the min./max. length for line 1 ;

- $\tau_{1}$ is the overall travel time for a generic network line;

- $\tau_{\min }$ and $\tau_{\max }$ are the min./max. travel time for line 1 ; $\tau^{l}$

- $\tau_{\max }$ is the max. acceptable deviation time of line 1; $\tau^{l}$

- $\tau_{\text {min }}$ represents the best path for line 1;

- $\quad \mathrm{W}$ is a max. deviation coefficient from the best path for line 1 ;

- $\quad \mathrm{D}_{\mathrm{i}, \mathrm{j}}{ }^{\mathrm{a}}$ is the distance between bus stop $\mathrm{j}$ and pedestrian node $\mathrm{i}$;

- $\quad D_{i, j}$ is the max. distance between bus stop $i$ and $j$;

- $\mathrm{D}_{\mathrm{a}}{ }^{\max }$ and $\mathrm{D}_{\mathrm{f}}^{\max }$ are the max. values for the previous distances;

- $\% \mathrm{P}$ is the percentage of direct demand to be served;

- $\quad$ P represents the total passenger flows on the public network;

- $f_{\min \text { and }} f_{\max }$ are the min./max. frequency on the network;

- $\tau_{g}^{l}$ is the total running time on 1 line;

- $\tau_{s}$ is the turn time;

- $\mathrm{T}_{\mathrm{m}}$ is the fare value from in mode $\mathrm{m}$ of $\mathrm{M}$;

- $t_{m}^{\operatorname{Min}} \mathrm{e}_{m}^{t_{m}^{\operatorname{Max}}}$ are the min./max. fare values in mode $\mathrm{m}$ of $\mathrm{M}$.

The first term of the objective function is the company's cost for a given network configuration, while the second characterizes the user's cost. Weights $\mathrm{C}_{1} \ldots \mathrm{C}_{6}$ were introduced in order to reflect the trade off between company and user. Usually, the element that affects immediately business management is the 
distance in kilometres covered by the vehicles which circulate within the given network. This was taken into account in the expression used by Bansal [13]. With regard to user's cost, five elements were considered: the walk time to reach the bus stop, the vehicle's wait time at the bus stop, the travel time on board, the fare paid to use that transportation mode and a non-comfort function expressed as the possibility to travel standing, [26]. The constraints can be classified into 3 types: geometric eqn.(2),..,eqn.(7), operative eqn.(8),..., eqn.(13), congruency eqn.(14),..., eqn.(16). Eqn. (2) considers the max. number of lines that can operate in a context. The limits to the max. and min. length of a line, and to its route running time have been included in eqn. (3) and (4); these constraints avoid the formation of lines that are either too long (thus needing numerous vehicles in order to ensure good frequency standards), or too short to obtain a reliable network. Eqn. (5) imposes a limit to the max. time of line deviation from the best path; eqn. (6) and eqn. (7), instead, impose limits to the max. network access distance from a pedestrian node and to the max. distance between bus stops. Usually, the models analyzed in the literature do not consider this factor. With regard to operative constraints, eqn.(8) represents the min. percentage of demand to be served directly; eqn.(9) imposes the frequency a lower bound below which the user would not benefit from the service, and an upper bound above which company costs would be too high. Eqn. (10) deals with the load factor so to guarantee that the max. flow on the critical link of every line cannot exceed the max. vehicle capacity for that line; eqn.(11) represents the resource limit for the company and guarantees that the optimal network models never use more vehicles than those available in the park; eqn. (12) considers the possibility to operate vehicles of various dimension on the network, while eqn. (13) determines the value of $\mathrm{min}$. and max. fares. Congruency constraints comprise those of flow conservation eqn. (14) and eqn. (15) and non-negative flow eqn. (16).

\subsection{Resolution process}

The resolution of the model should, theoretically, take place with the use of a global method. However, because of the non-linear and combinatorial nature of the problem, this formulation appears inappropriate [16, 26, 29]. Moreover, the proposed model is multi-objective, non-linear and is characterised both by integer variables (e.g. the number of buses that circulate in a peak hour) and continuous variables (e.g. the passenger flows on a line). Hence, its resolution requires the implementation of heuristic techniques, which can lead to a successful solution of the problem. However, due to the unreliability of some of the variables, it is more appropriate to refer to an optimal area rather than a single optimal solution. Therefore, it is necessary to solve the problem by breaking it down into several parts. The first is represented by a heuristic greedy technique, which generates a desirable set of routes. At this stage, the frequencies and the elements directly connected to them are not considered while, in the construction of the base routes, particular attention is paid to those geometric constraints related to the characteristics of the service. In the second part is possible to optimize (through a random search) the optimal frequencies 
for every line and to introduce those constraints omitted in the first phase. The number of lines to be optimized is determined through three phases: input data acquisition and initialisation; elaboration; route network restitution. Phase 1 deals with the acquisition of supply and demand data. With regard to supply data, it is necessary to identify the nodes that cause greater delay to the circulation and those likely to generate demand thanks to their proximity to schools, public offices, etc. When analyzing the demand data, it is necessary to acquire the matrices related to: the demand, the distance between centroids and the passenger- $\mathrm{km}$. Phase 2 represents the core of the method since it is linked to all the elaborations necessary to build the network. The demand matrix is cleared from the O/D pairs which represent null journeys, and for each pair the min. cost path is created using an efficient algorithm, [30], previously modified. Once the min. path cost for every O/D pair (calculated both for the forward and backward paths) is known, the 3 matrices previously defined are rearranged in decreasing order. Consequently, the matrices are examined individually and three different networks are created, one for each typology of matrix. The algorithm steps are:

Step 1. (Common to all the ordering strategies). For each OD pair the min. direct and alternative routes are created introducing, in the latter case, some penalties or bonuses if the route crosses nodes characterized by remarkable time losses or nodes located near areas with high volumes of traffic.

Step 2. The O/D matrix is arranged in decreasing order according to transport demand.

Step 3. The OD pair of highest value is extracted from the demand matrix.

Step 4. That pair is eliminated from the matrix.

Step 5. The route connecting the pair is extracted from the min. paths list.

Step 6. A line of public transport is associated to the min. route.

Step 7. If the line serves other O/D pairs (directly or within a reasonable pedestrian distance -eqn. (6), and therefore contains a number of sub-routes, the pairs served are eliminated from the matrix and are considered satisfied from the line; otherwise, step 8 follows.

Step $8 . \quad$ The list of direct routes is updated.

Step 9. If the initial matrix is empty or if a certain percentage of fixed demand -eqn.(8)- has been satisfied, step 10 follows; otherwise the procedure goes back to step 2 .

Step 10. The initial matrix is recalled and recharged.

Step 11. The O/D pair, which displays the highest value, is extracted from the matrix demand.

Step 12. The abovementioned pair is eliminated from the matrix.

Step 13. The alternative route is extracted from the list of the alternative routes.

Step 14. If the travel time exceeds a predetermined min. time threshold the line is rejected and the procedure goes back to step 11. Otherwise step 15 goes on.

Step 15. If the alternative line serves other O/D pairs (directly or within a reasonable pedestrian distance -eqn. (6), and therefore contains a number of 
sub-routes, the pairs served are eliminated from the matrix and are considered satisfied from the line; otherwise, step 16 follows.

Step 16. The list of the alternative routes is updated.

Step 17. If the initial matrix is empty the procedure goes to the next step; otherwise it returns to step 11.

Step 18. The lists of the direct and alternative routes are confronted in pairs and on the basis of an inner function objective, so to verify the presence or absence of $\mathrm{O} / \mathrm{D}$ terminal nodes served either by the direct route or the alternative one or both.

Step 19. A new list is updated. This list is obtained by inserting either direct lines (if not present in the list of the alternative lines) or those obtained by means of a comparison.

Step 20. If all the direct and alternative lines have been examined, step 21 follows; otherwise the procedure goes back to step 18 .

Step 21. The scheme of lines obtained ordering the demand matrix is made definitive.

The distance and pass*km matrices are calculated in the same way. Once the described algorithms are determined, phase 3 begins. Networks are confronted according to the value assumed by an objective function included in the procedure (see 2.2.1 and 2.2.2). The result of this comparison supplies the base network (including the bus stops).

\subsubsection{Construction of base scenery}

Step 1. The lists of routes which characterize the three scenarios are recalled.

Step 2. The lists of the first two scenarios are compared in pairs and on the basis of an inner objective function to minimize.

Step 3. A scenario comprised of a selection of the above is identified.

Step 4. The lists of the last defined scenario and that resulting from the previous comparison are confronted in pairs and on the basis of an inner objective function to minimize.

Step 5. A scenario based on the best of these and formed by a list of lines that constitute the base scenarios on which to continue the search is identified.

\subsubsection{Extraction of the final scenario}

Step 1. The first line is extracted from the list.

Step 2. Length and time constraints are verified. If results are positive, the line is inserted in the final configuration on which to optimize the frequencies. Otherwise, it is eliminated and the procedure goes back to step 1.

Step 3. The line is inserted in the final scenario;

Step 4. Eqn. (2) is checked. If results are positive, the procedure goes back to step 1. Otherwise it proceeds.

Step 5. The set of the lines on which to optimize the frequencies is made definitive.

Step 6. The bus stops for the better lines are positioned respecting eqn. (7). 
Once the final scenario is defined, formed at the most by ML lines, it is possible to proceed with the determination of the frequency values to assign to the lines. Once the routes are defined and assuming that: a) the fare is the same for the m modes and within a given mode, so to consider it a constant value and therefore negligible and b) the company owns vehicles of one dimension only, so that the in-vehicle time is unaffected by the vehicle dimension it is possible to proceed to the determination of the frequencies. This is possible through the simplification of the non-linear model by applying a random search procedure. Indeed, starting with a set of initial frequencies and characterized a method of improvement through some objective criterion, a new, improved, solution is reached. In short, initial hyper-paths are assigned to determine passenger flows on the lines. Then, a further frequency improvement is sought by identifying a global search direction which originates from the same lines competing on the number of passengers. For those lines that present higher user flows, the frequency is increased, while it is reduced for those with a lesser load. Subsequently, based on these indications, further frequencies are assigned to the lines to determine new flows and to be able to recalculate the value of the already defined objective function. If the values differ on a fixed a priori convergence parameter, the value of the line frequencies found can be considered acceptable. Otherwise a new iteration of the process becomes necessary until the convergence is reached. The steps of the algorithm are summarised below.

Step 1. A stop parameter is fixed.

Step 2. A set of frequencies is initialized for all the lines, while respecting eqn. (9) and eqn. (10). In relation to eqn. (9), integer values easily memorisable by the user (practical approach), are used.

Step 3. Hyper-paths are assigned to the transportation network, so to calculate the passenger flows and to verify the respect of eqn. (10).

Step 4. The value of the objective function - eqn. (1) - is calculated.

Step 5. A new search direction is sought by evaluating the most and least loaded lines.

Step 6. The new frequencies are reassigned respecting eqn. (9) and eqn.

Step $7 . \quad$ See step no.3.

Step 8. $\quad$ See step no. 4.

Step 9. The convergence test is carried out. If verified, the algorithm stops and provides the set of optimal frequencies, otherwise the procedure returns to step 5 .

\subsection{IT instruments used}

The model has been created through a complex JAVA language application. The phase that brings to the construction of the basic scenario of the network lines has been fully developed while the second phase is ongoing. However, in order to carry out some tests and compare such planning procedure with the present network, an external application has been used to provide the assignment results. These, enable the calculation of the objective function. 


\section{Model application in a real network of a medium dimension city}

The proposed model has been tested on a medium-sized city in Sardinia (Italy). A number of demand and supply data necessary to model the case study context have been acquired, and an extended transportation network has been modelled. A network portion has been extracted from this network (Quartu Sant'Elena; 70.000 inhabitants). Such network presents: 22 traffic zones, 855 nodes (22 centroids), 1414 links (62 fictitious, 495 one - way links and 857 two - way links). Subsequently, the localization of the main public transport generation points was carried out with GIS instruments, together with the identification of the main junctions that can affect traffic flows. With regard to the main traffic attractors, it was considered appropriate to identify the following buildings categories: schools, centres for young and elder people, banks, post offices and other buildings. Each of these items has been located on a map and has been described with the following attributes: a numerical code, a name that briefly describes it, address, type, a numerical value to be used in path construction and the nearest node to the building. With regard to the numerical value, an attempt was made to attribute the best values to those attractors that are easier to reach via public transport. Main junction nodes have been modelled according to the location of traffic lights on the map. Every junction has been described via: a numerical code; an address location; the traffic type at the given junction; a penalty that represents a numerical value, quantified according to the traffic volume and to be used in the path construction; a node that denotes the network junction. The demand matrix has been acquired from a previous study [31] and its main characteristics highlight that: it refers to a scholastic week day winter service, between 7,00 and $8,00 \mathrm{am}$, it includes only public transport journeys for study and work reasons.

\subsection{Experimental results}

Once the supply and demand data input have been acquired, it has been possible to apply the proposed planning model to the city network. In particular, the nonnull O/D pairs have been initially selected. Subsequently, the min. direct and alternative paths have been constructed for the three matrices using the defined penalties. Subsequently, the list of direct and alternative lines has been created, resulting in a scenario that comprised 33 lines, reduced to 17 once length and time constraints were imposed (3 less than in the current scenario). Starting from this configuration, the definitive lines have been associated to the bus stops, and the service frequencies could be calculated on the number of vehicles currently used. Indeed, an iterative process for the frequency calculations has been applied. From the analysis, it emerges that the line length is significantly reduced. This can be due to the routes, which could be on the min. road distance. In addition, the service intervals (easily recognisable by the users) correspond to entire frequencies. The model converges quickly and this can be justified by the lower values of the transportation demand, which do not contribute meaningfully to a more limited reduction of the objective function. 


\subsection{Some considerations}

On the basis of the experimental results, some considerations on the goodness of the solution can be drawn. It can be argued that the methodology results seem satisfactory, even though a more meaningful judgment could be given once further parameters are better calibrated and refined and the model behaviour is evaluated for a complete demand matrix. Moreover, since the testing phase is still in process, the control parameters are being calibrated in order to carry out the sensitivity tests. Even so, it has been possible to carry out a comparison with the existing network.

\section{Comparison with existing network}

The existing public transport system in Quartu S.E. has been considered isolated and independent from the large Cagliari's public transportation network. In fact, the city of Quartu S.E. is served currently from CTM lines and it does not benefit from a public transport network of its own. Therefore, the lines, that leaving Cagliari cross the city, have been broken and only the part inside Quartu S.E. was considered. Currently, several lines present service intervals whose presence is not appreciated by the users (e.g. 1 bus/hour) while in other lines some of the values are not easy to memorise. Therefore, the new and the existing networks were compared so to verify the validity of the method. The comparison was made calculating the index impact (e.g. efficiency, effectiveness and quality). These assignments have been performed using a simulation software (CubeTRIPS $^{\mathcal{O}}$ ) and, subsequently, proceeding to the index calculation.

\subsection{Impact index based comparison}

The efficiency indexes used (see tab. 1) are: line numbers (L), total network length $\left(\mathrm{L}_{1}\right)$, total travel time $\left(\mathrm{T}_{1}\right)$, average speed $\left(\mathrm{V}_{\mathrm{m}}\right)$ and bus size $(\mathrm{B})$. The effectiveness indexes used are: on-board passengers $(\mathrm{U})$, users / vehicle* $\mathrm{km}$ $(\mathrm{U} / \mathrm{VKM})$, users/network length $(\mathrm{U} / \mathrm{L})$ and users* $\mathrm{km} /$ vehicle * $\mathrm{km}$ (UKM/VKM) ratios. The quality indexes used are: total and weighed walk time $\left(\mathrm{T}_{\mathrm{p}}, \mathrm{T}_{\mathrm{p}}{ }^{\mathrm{OD}}\right)$, in-vehicle time $\left(\mathrm{T}_{\mathrm{b}}, \mathrm{T}_{\mathrm{b}}{ }^{\mathrm{OD}}\right)$, wait time $\left(\mathrm{T}_{\mathrm{a}}, \mathrm{T}_{\mathrm{a}}{ }^{\mathrm{OD}}\right)$ and boarding. In the case of the efficiency indexes, the solution project appears to be better than the existing one because, for a fixed bus size, it presents: a shorter distance, a shorter travel time and higher speed. Therefore, the applied procedure has proved to be good enough for the company objectives. In the case of effectiveness indexes, the line network project is better than the existing one because: the number of boarding passengers is greater and therefore there are less empty seats on the bus; the lines are shorter and consequently the vehicle/kilometres ratio is smaller. At the same time, the last index shows better values for the planned scenario compared to the existing situation. The quality indexes show that the walk times in the network project are better than in the current situation. Wait and in-vehicle time are slightly better in the existing network than in the new one but, with regard to the overall travel time, the network project is favourable. The better 
results obtained for the existing network are due to a shorter in-vehicle travel time, while with regard to the wait time, two explanations can be given. The existing network is supply-oriented because some corridors are transited by many lines, while others are barely catered for. Moreover, as a result of the network structure, users prefer to walk to the network access points along those corridors served by more attractive lines (therefore shortening wait time but increasing walking distance). In the network project (demand based), the walking distance is shorter since the bus stops are localized near zones characterized by demand mobility.

Table 1: $\quad$ Index comparison.

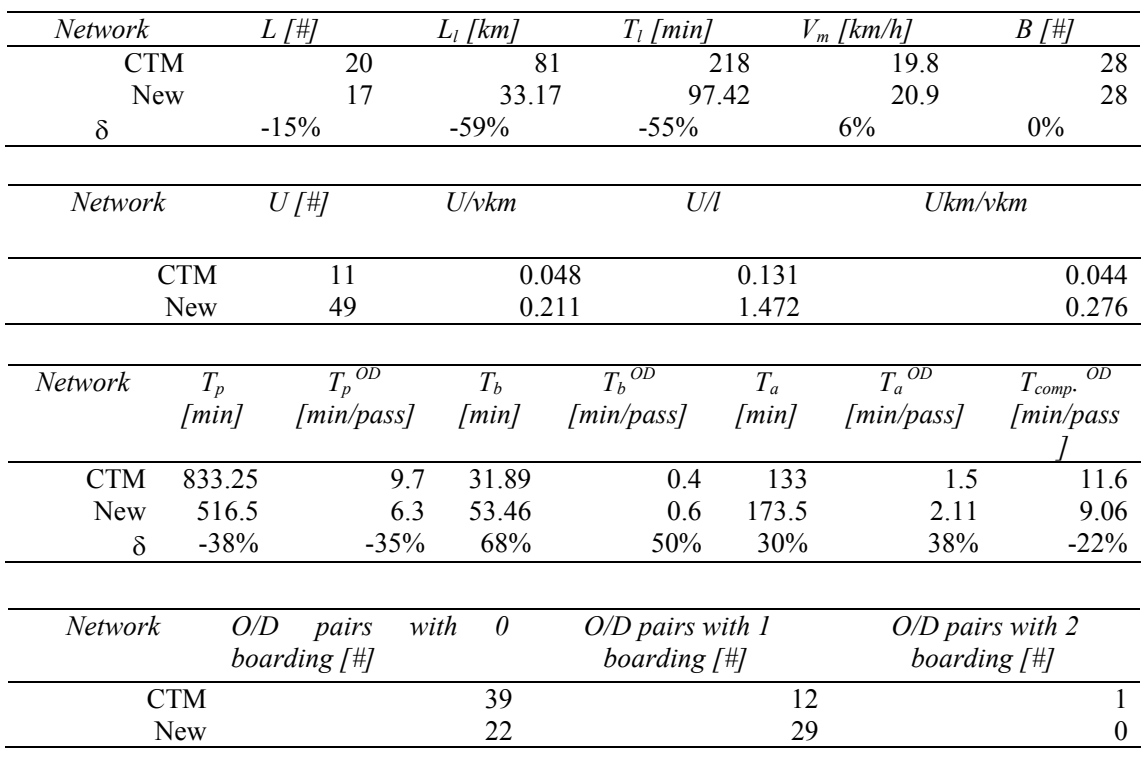

Finally, the last rows of table 1 show the number of $\mathrm{O} / \mathrm{D}$ pairs served by 0,1 and 2 boarding for the existing and project networks. Also in this case, the solution project is favourable because the same number of $\mathrm{O} / \mathrm{D}$ pairs is served with no more than a single boarding, so to satisfy the demand completely.

\section{Conclusions}

This paper has dealt with a TBRND and has proposed a new heuristic model for its resolution. In particular, it has described this complex problem examining different solution methodologies that have been categorised into empiric, what if and what to. Therefore, a project model defining a complex objective function has been structured in order to determine routes and frequencies. For its resolution, a complex heuristic algorithm has been used, which can be divided in two phases. In the first one, the route lines are characterized whilst, in the second, the frequencies are determined, respecting geometric, operative and 
congruence constraints. Furthermore, greedy resolution techniques were employed in the first part, while the second part of the algorithm has been resolved through a random search. The method was implemented and resolved using JAVA language. This model has been applied to a medium-sized city in Italy, and it has provided some interesting results which make the adopted procedure valid in comparison to the existing network. Results proved effective with specific regard to the business service, standing out for shorter distances covered by the lines and a $22 \%$ reduction in the overall travel time. However, the available demand data were restricted and might have affected the results positively. Indeed, the convergence was purposefully reached after a reduced number of iterations, mostly caused by the limited data available. The results, whilst confirming the proposed model, allow room for more detailed research. In particular: a new calibration of the objective function weights appears to be necessary in order to carry out the assignment procedure; the limit values to be assigned to the constraints need further calibration in order to identify those more appropriate to the case study; such methodology is to be extended to a more complex network and to more complete demand data; the possibility to use an agent language for the system modelling and speed up the procedure automatism is to be investigated.

\section{References}

[1] Scheidegger, P., Marchand, L., Delva G., Quidort M., Vivier J. \& Allen, H., Better Mobility in urban Areas. Problems, Solutions, Best Practices, UITP, pp. 1-48, 2001.

[2] Lampkin, W. \& Saalmans. P. D., The design of Routes, Service Frequencies, and Schedules for a Municipal Bus Undertaking. A case Study. Operational Research Quarterly, 18(4), pp. 375-397, 1967.

[3] Ceder A \& Wilson N. H. M., Bus network design, Transportation Research B, 20(4), pp. 331-344, 1986.

[4] Axhausen K. W. \& Smith, Jr. R. L., Evaluation of heuristic transit network optimization algorithms, Transportation Research Record, 976, pp.7-20, 1984.

[5] Chua, T.A., The planning of urban bus route and frequencies: a survey, Transportation, 12(2), pp.147-172, 1984.

[6] Russo, F., Musolino, G. \& Velonà, P., Modelli e metodi per il progetto dei sistemi di trasporto collettivo. Uno stato dell'arte. Modelli e Metodi per la programmazione di servizi di trasporto pubblico locale: uno stato dell'arte, ed. F. Russo, Franco Angeli: Milano, pp. 37-53, 2001.

[7] AA., VV., Bus route and schedule planning guidelines, Transportation Research Board: Washington, DC, pp.1-99, 1980.

[8] Pratt, R. H. \& Schultz, G. W., A systems approach to sub-area transit service design, Transportation Research Record 419, pp.37-471, 1972.

[9] O' Brien, W.B., An example of the functional design and costing of transit routes, Urban Bus Transit-a planning guide, ed. 2J. H. Shortreed, University of Waterloo: Canada, 1974. 
[10] Byrne B. F. \&Vuchic F. C., Public Transportation line position and headways for minimum cost, Proceedings of the $5^{\text {th }}$ international symposium on theory of road traffic flow and Transportation, American Elsevier: New York, pp. 347-360, 1972.

[11] Kikuchi, S., Relationships between the Number of Stop and Headway for a Fixed Route Transit System, Transportation Research A, 19(1), pp. 6571, 1985.

[12] Baaj, M. H., Mahmassani, H. S., Hibrid route generation heuristic algorithm for the design of transit networks, Transportation Research $C$, 3(1), pp. 31-50, 1995.

[13] Bansal, A. N., Optimization of bus route network for fixed spatial distribution, Scientific Management of transport system, ed. N.K. Jaiswall, North Holland Publishing Company, The Netherlands, pp.346355, 1981.

[14] Bielli, M., Caramia, M. \& Carotenuto, P., Genetic algorithms in bus network optimization, Transportation research $C, \mathbf{3 ( 6 )}$, pp.445-463, 2002.

[15] Carrese, S. \& Gori, S., An urban bus network design procedure (Chapter 11), Transportation Planning-State of the Art, eds. M. Patriksson \& M. Labbe, Kluwer Academic Publisher, pp. 177-195, 2002.

[16] Chien. S., Yang, Z. \& Hou, E., Genetic Algorithm for Transit Route Planning and Design, Journal of Transportation Engineering, 127(3), pp. $200-207,2001$.

[17] Constantin, I. \& Florian, M., Optimizing. Frequencies in a Transit Network: a Nonlinear Bi-Level Programming Approach, International Transportation in Operational Research, 2(2), pp. 149-164, 1995.

[18] Fan, W. \& Machemehl, R. B., A Tabu Search Based Heuristic Method for the Transit Route Network Design Problem, Paper presented at $9^{\text {th }}$ International Conference on Computer-Aided Scheduling of Public Transport (CASPT), August 9-11, San Diego, California, 2004.

[19] Fan, W. \& Machemehl, R.B., Using a Simulated Annealing Algorithm to Solve the Transit Route Network Design Problem, Journal of Transportation Engineering, 132(2), pp. 122-132, 2006.

[20] Furth, P. G. \& Wilson N. H. M., Setting frequencies on bus routes: theory and practice, Transportation Research Record, 818, pp.1-7, 1981.

[21] Mandl, C. E., Evaluation and optimization of urban public transportation networks, European Journal of Operational Research, 5(6), pp. 396-404, 1980.

[22] Montella B., Gallo G. \& Amirante R., A general bus network design model and its application, Paper presented at TRISTAN III, San Juan, Puerto Rico, 1998.

[23] Pattnaik S. B., Mohan S. \& Tom, V. M., Urban Bus Route network design using genetic algorithm, Journal of Transportation Engineering, 124(4), pp. 368-375, 1998. 
[24] Quak, C. B., Bus line Planning. A passenger-oriented approach of the construction of a global line network and an efficient timetable, Degree thesis, Delft University of Technology, Netherlands, 2003.

[25] Rea, J., Designing Urban Transit System: an Approach to the Route Technology Selection Problem, Highway Research Record, 417, pp. 4859, 1972.

[26] Silman, L.A., Barzily, Z., \& Passy, U., Planning the Route System for Urban Bus, Computers and Operational Research, 1(2), pp. 201-211, 1974.

[27] Van Nes, R., Hamerslag. R. \& Immers H. B., Design of public Transport Networks, Transportation Research Record, 1202, pp. 74-83, 1988.

[28] Nguyen, S, \& Pallottino, S., Hyperpaths and shortest hyperpaths, Combinatorial Optimization Lecture Notes in Mathematics, 1403, pp. 258-271, 1989.

[29] Chien, S., \& Yang, Z., Optimal Feeder bus Routes on Irregular Street Networks, Journal of Advanced Transportation, 34(2), pp. 213-248, 2000.

[30] Dijkstra, E. W., A note on two problems in connexion with graphs, Numerische Mathematik, 1, pp. 269-271, 1959.

[31] Metis Consulting, Piano di riordino della rete dei servizi di trasporto pubblico locale. Prepared for CTM, 1998. 